\title{
ERK2 CD domain mutation from a human cancer cell line enhanced anchorage-independent cell growth and abnormality in Drosophila
}

\author{
MARTHANDAN MAHALINGAM ${ }^{1}$, RAMANATHAN ARVIND $^{1^{*}}$, HIROYUKI IDA $^{2^{*}}$, \\ AVANIYAPURAM KANNAN MURUGAN ${ }^{1}$, MASAMITSU YAMAGUCHI $^{2}$ and NOBUO TSUCHIDA ${ }^{1}$ \\ ${ }^{1}$ Department of Molecular Cellular Oncology and Microbiology, Tokyo Medical and \\ Dental University, Tokyo 113-8549; ${ }^{2}$ Department of Applied Biology and Insect \\ Biomedical Research Center, Kyoto Institute of Technology, Kyoto 606-8585, Japan
}

Received April 14, 2008; Accepted May 26, 2008

DOI: 10.3892/or_00000096

\begin{abstract}
In a human cancer cell line, we previously found a mutation in codon 322 of the extracellular signal-regulated kinase $\left(E R K 2^{E 322 K}\right)$, the protein showed a faster migration when compared to wild-type in SDS-PAGE and constitutive phosphorylation. However, the reason for the faster migration, and the biochemical and biological properties of the mutation is unknown. In this study, we report that the amino acid charge-change mutation in the common docking (CD) domain is important for fast migration. In vitro binding of ERK $2^{\mathrm{E} 322 \mathrm{~K}}$ to MKP1 and RSK2 was lost, resulting in constitutive activation and possibly contributing to a more efficient colony formation in soft agar. We established transgenic flies by carrying the corresponding CD domain mutation, DERK ${ }^{E 335 K}$, which developed smaller and rougher eyes compared with the wild-type. Taken together, these data are consistent with ERK2 ${ }^{\mathrm{E} 322 \mathrm{~K}}$ loss of contact with downstream effectors and its constitutive activation, presenting an oncogenic potential and weak abnormality in differentiation.
\end{abstract}

\section{Introduction}

The extracellular signal-regulated kinase (ERK) belongs to the mitogen-activated protein kinase (MAPK) family, which plays important roles in cell growth, differentiation, and

Correspondence to: Dr Nobuo Tsuchida, Department of Molecular Cellular Oncology and Microbiology, Tokyo Medical and Dental University, 1-5-45 Yushima, Bunkyo-ku, Tokyo 113-8549, Japan

E-mail: tsuchida.mcom@tmd.ac.jp

${ }^{*}$ Contributed equally

Key words: extracellular signal-regulated kinase 2, mitogenactivated protein kinase, MKP, transformation, sevenmaker, common docking domain survival $(1,2)$. ERK is activated by extracellular growth factors through RTKs (3). ERK is up-regulated with the upstream dual kinase, MEK (4), which phosphorylates threonine and tyrosine residues of the TEY sequence in ERK (5). In contrast, ERK is down-regulated with ERK-regulated phophatases with dual-specificity phosphatase activity, keeping ERK activation under control (2). Activated ERK activates RSK in the cytoplasm, enters the nucleus and phosphorylates various transcriptional factors such as ELK-1 (6) through phosphorylation at serine/threonine residues of the target proteins (7).

It has been reported that various signal transductions are mediated through the acidic amino-acid rich common docking (CD) domain. A gain-of-function mutant within this region was first reported in sevenmaker of Drosophila (DERK $\left.{ }^{\text {D334N/sem }}\right)$ (8), resulting in loss of binding to MKP1 $(9,10)$ and RSK $(11)$.

We previously reported a CD domain mutant, ERK2 $2^{\mathrm{E} 322 \mathrm{~K}}$, from a human cancer cell line that was detected as a fastermigrating band in SDS-PAGE (12). Herein, we examined the biochemical and biological properties of the faster-migrating ERK $2^{\mathrm{E} 322 \mathrm{~K}}$ and found that other amino acid charge-change mutations at other sites in the CD domain, including human counterparts of ERK ${ }^{\mathrm{sem} / \mathrm{D} 321 \mathrm{~N}}$, resulted in faster migration. $E R K^{E 322 K}$ induced the anchorage-independent growth of Balb3T3 cells in soft agar and weak abnormality in Drosophila eye development.

\section{Materials and methods}

Cell lines and culture. HaCaT (13), HEK 293T, HSC6, Ca922, Saos-2 and Balb3T3 cells were cultured in complete Dulbecco's modified Eagle's medium (DMEM) (Nissui Pharmaceuticals, Tokyo, Japan) and supplemented with $10 \%$ FBS (JRH Biosciences, Tokyo, Japan) under humidified 5\% $\mathrm{CO}_{2} / 95 \%$ air.

Construction of HA-tagged and LTR plasmids. ERK2 ${ }^{\mathrm{wt}}$ or ERK $2^{\mathrm{E} 322 \mathrm{~K}}$, originally cloned in $\mathrm{pGEM}^{\circledR}-\mathrm{T}$ easy vector (Promega, Madison, WI), were PCR-amplified using 5'-TTG ATATCTTATGGCGGCGGCG-3' (forward) and 5'-GGCTC GAGTTAAGATCTGTATCCTGGCTG-3' (reverse) primers, 
digested with EcoRV and XhoI and cloned in-frame in pcDNA3-2HA. Two tandem copies of hemeagglutinin epitope sequences were inserted at HindIII and EcoRI sites.

To express in mouse cells the NotI fragment of ERK2, originally cloned in pGEM-T easy vector, the cells were cloned in a pLTR-SA vector (14) where the BamHI site was changed to NotI site by blunting the BamHI site followed by Not I linker ligation. A neomycin derivative (G418) gene was inserted.

Plasmid purification was performed using an endofree DNA purification kit (Qiagen Japan, Tokyo).

Site-directed mutagenesis. In vitro mutagenesis of ERK2 was performed as described in the QuickChange ${ }^{\circledR}$ site-directed mutagenesis kit (Stratagene, La Jolla) using the primer sets: D321K/5'-ATTACGACCCGAGTAAAGAGCCCATC-3' (forward) and 5'-GATGGGCTCTTTACTCGGGTCGTAAT-3' (reverse), K321N/5'-CGACCCGAGTAACGAGCCCATCG CCG-3' (forward) and 5'-CGGCGATGGGCTCGTTACTC GGGTCG-3' (reverse), D318K/5'-CTGGAGCAGTATTA CAAACCGAGT-3' (forward) and 5'-ACTCGGTTTGTAA TACTGCTCCAG-3' (reverse), E250K/5'-TGGATCCCCA TCACAAAAAGACCTGAATTG-3' (forward) and 5'-CAAT TCAGGTCTTTTTGTGATGGGGATCCA-3' (reverse), and M13L/5'-GCGGGCCCGGAGCTGGTCCGCGGGCAGG-3' (forward) and 3'-CCTGCCCGCGGACCAGCTCCGGGCC CGC-3' (reverse). The mutations were introduced in HAERK2 $^{\text {wt }}$. D321N was constructed from D321K. The mutant constructs were confirmed by sequencing (ABI PRISM version 1, ABI Japan, Tokyo).

Treatment with EGF and mammalian cell lysate preparation. Ca922 cells were serum-starved in DMEM for two days, and stimulated with EGF (10 ng/ml, Sigma-Aldrich, MO) for $1 \mathrm{~h}$. Cells were then washed with ice-cold PBS and lysed in NP-40 lysis-buffer as previously described (12). Nuclear extracts were prepared from Saos-2 cells as previously described (15).

Western blotting. Western blotting was performed as previously described (16). Anti-MKP1, anti-ERK2 (D-2), anti-ERK1 (C-16) and anti-RSK2 (C-19) (Santa Cruz, CA), as well as anti-HA (Boehringer Mannheim, Germany) and the HRP conjugates (New England Biolabs) were used.

GST-tagged vector, bacterial growth and lysate preparation, and pulldown assay. GST-ERK2 ${ }^{\mathrm{wt}}$ and GST-ERK2 ${ }^{\mathrm{E} 322 \mathrm{~K}}$ were constructed (17), and used to transform E. coli BL21 (DE3), which was induced and lysed as previously described (16). GST-ERK2 ${ }^{\mathrm{wt}}$, GST-ERK2 ${ }^{\mathrm{E} 322 \mathrm{~K}}$ and GST-vector containing bacterial lysates were used for the pulldown assay (18) of MKP1 and RSK2 proteins in Ca922 total cell and Saos-2 nuclear lysates.

Colony formation assay in soft agar. Balb3T3 cells were lipofected (LF2000, Invitrogen) by using the LTR-ERK2 ${ }^{\mathrm{wt}}$ or -ERK2 ${ }^{\mathrm{E} 322 \mathrm{~K}}$ or -vector alone according to the manufacturer's directions, and grown with $400 \mu \mathrm{g} / \mathrm{ml} \mathrm{G} 418$. For clones stably expressing the wild-type or mutant proteins, the cells were left with G418 for three weeks. The cell colonies were isolated by using cloning cylinders and grown separately with

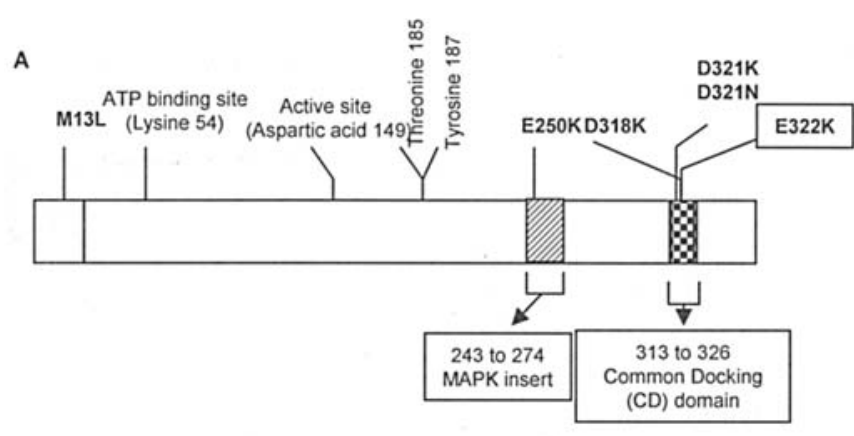

B

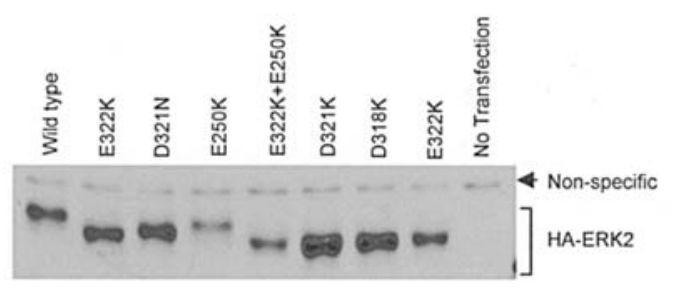

C

D
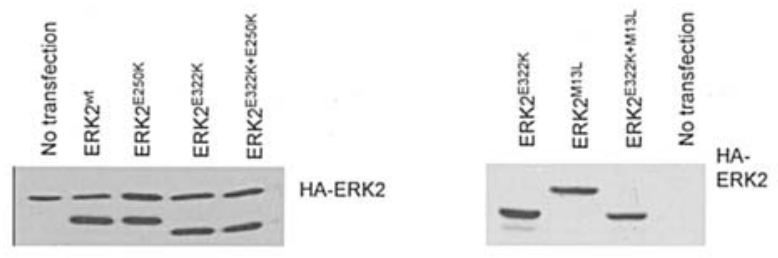

Figure 1. (A) Schematic illustration of the functional domains (sites) in human ERK2, in which the mutants constructed are shown in bold letters. (B) Various mutants in the CD domain of ERK2 proteins with amino acid charge-change migrated faster in SDS-PAGE. 293T cells were transiently transfected with one of the HA-tagged various mutant genes or with HAERK2 $2^{\text {wt }}$. Cell lysates (equal amount of proteins) were electrophoresed in $12 \%$ SDS-PAGE, and then Western-blotted using anti-HA antibody. (C) Migration of the ERK2 ${ }^{\mathrm{E} 322 \mathrm{~K}+\mathrm{E} 250 \mathrm{~K}}$ double mutant in SDS-PAGE. 293T cells were transfected with HA-tagged ERK2 ${ }^{\text {wt }}$, ERK2 ${ }^{\mathrm{E} 322 \mathrm{~K}}$, ERK2 ${ }^{\mathrm{E} 250 \mathrm{~K}}$ and the double mutant ERK2 ${ }^{\mathrm{E} 322 \mathrm{~K}+\mathrm{E} 250 \mathrm{~K}} \mathrm{DNA}$ and Western-blotted using anti-HA antibody. (D) Migration of ERK2 ${ }^{\mathrm{M} 13 \mathrm{~L}}$ in SDS-PAGE. 293T cells were transfected with HA-tagged ERK2 ${ }^{\mathrm{M} 13 \mathrm{~L}}, \mathrm{ERK} 2^{\mathrm{E} 322 \mathrm{~K}}$ and ERK2 ${ }^{\mathrm{M} 13 \mathrm{~L}+\mathrm{E} 322 \mathrm{~K}}$ DNA and Western-blotted using anti-HA antibody.

fresh complete medium. The clones positively expressing ERK2 $^{\text {wt }}$ were identified by using anti-ERK1 (C-16) antibody, which predominantly recognizes human ERK1 and to a lesser extent human ERK2. Mutant ERK2 ${ }^{\mathrm{E} 322 \mathrm{~K}}$ was identified by its faster-migrating property. For cells transiently expressing wild-type or mutant ERKs or just the vector, cells were grown for 2 days in the presence of G418 and used for the soft agar assay. A bottom agar in DMEM with $0.5 \%$ agarose and top agar with 3000 cells and $400 \mu \mathrm{g} / \mathrm{ml} \mathrm{G} 418$ in DMEM and $0.33 \%$ agarose were made, incubated in $5 \% \mathrm{CO}_{2}$ and monitored for colony formation as previously described (17), with a few modifications.

Preparation of Drosophila cDNA. Total RNA was isolated using a TRIzol ${ }^{\circledR}$ reagent (Invitrogen) and cDNA was prepared with an AgPath-ID ${ }^{\text {TM }}$ one-step RT-PCR kit (ABI) as described in the respective kits. 
pUAST-DERK ${ }^{E 335 K}$ plasmid construction. pUAST (19) is a P-element vector (pCaSpeR3) containing the P3' and P5' ends of $\mathrm{P}$ element and the white gene which acts as a selection marker for successful integration into the Drosophila genome. To construct the pUAST-DERK ${ }^{\mathrm{wt}}$ and pUAST-DERK ${ }^{\mathrm{E} 335 \mathrm{~K}}$ plasmids, Drosophila ERK cDNA was amplified by using 5'-TAGCGGCCGCATGGAGGAATTTAATTCG-3' (forward, with NotI site) and 5'-AACTCGAGTTAAGGCGCATTGTC TGG-3' (reverse, with XhoI site) primers, and amplicon was inserted into pGEM-T easy vector. The clones were PCRamplified using the above-mentioned forward and 5'-AAGT CGACTTAAGGCGCATTGTCTGG-3' (reverse, with SalI site) primers and cloned into the pUAST vector. Then, in vitro mutagenesis was performed using 5'-GATCCTGGAGATAA GCCTGTCGCTGAAG-3' (forward) and 5'-CTTCAGCGA CAGGCTTATCTCCAGGATC-3' (reverse) primers and the mutagenized sequence was confirmed by sequencing.

Establishment of transgenic flies. The plasmids pUASTDERK $^{\text {wt }}$ or -DERK ${ }^{\mathrm{E} 335 \mathrm{~K}}$ or the empty vector were injected in fertilized Drosophila eggs and the P-element-mediated germ line transformation was carried out as described earlier (20). F1 transformants were selected on the basis of white eye-color rescue (21). The established transgenic flies were crossed with the transgenic fly line (number 16) carrying GMR-GAL4 (22).

Scanning electron microscopy. Adult transgenic flies were anesthetized, mounted on stages and observed with a VE-7800 scanning electron microscope (Keyence Inc., Osaka, Japan) in a high vacuum mode.

Statistical analysis. A comparison between the numbers of colonies formed by wild-type or mutant ERKs in soft agar was made with unpaired Student's t-test for continuous outcomes. P-values were two-sided and $\mathrm{p}<0.05$ was considered statistically significant.

\section{Results}

ERK2 proteins with amino acid charge-change mutations in the $C D$ domain migrate faster in SDS-PAGE. Our earlier study revealed that the faster migrating mutant ERK2 ${ }^{\mathrm{E} 322 \mathrm{~K}}$, found in the HSC6 cancer cell line (12), was $40 \mathrm{kDa}$. Since the protein was detected with a C-terminal anti-ERK2-specific antibody, we excluded the presence of premature termination as the reason for faster migration. A possibility is the presence of an internal start site at codon 13, resulting in a constructed HA-ERK2 $2^{\mathrm{M} 13 \mathrm{~L}}$, which was examined for migration, while another possibility is the amino acid charge-change mutation in the CD domain or in the MAPK insert region. Thus, we constructed other HA-tagged mutants in the CD domain (23) and MAPK insert region (24) as shown in Fig. 1A.

293 T cells transiently expressing HA-ERK2 mutants showed that charge-change mutation in the MAPK insert region $\left(\mathrm{ERK} 2^{\mathrm{E} 250 \mathrm{~K}}\right)$ did not markedly affect its migration in SDS-PAGE. However, mutants in the CD domain (ERK2 ${ }^{\mathrm{E} 322 \mathrm{~K}}$, ERK2 ${ }^{\mathrm{D} 321 \mathrm{~N}}$ and ERK2 ${ }^{\mathrm{D} 318 \mathrm{~K}}$ ) resulted in a faster migration (Fig. 1B). In addition, the double mutant (ERK2 ${ }^{\mathrm{E} 322 \mathrm{~K}+\mathrm{E} 250 \mathrm{~K}}$ ) migrated as ERK2 $2^{\mathrm{E} 322 \mathrm{~K}}$ but not as ERK2 ${ }^{\mathrm{E} 250 \mathrm{~K}}$ (Fig. 1C), confirming that the amino acid charge-change mutation in the

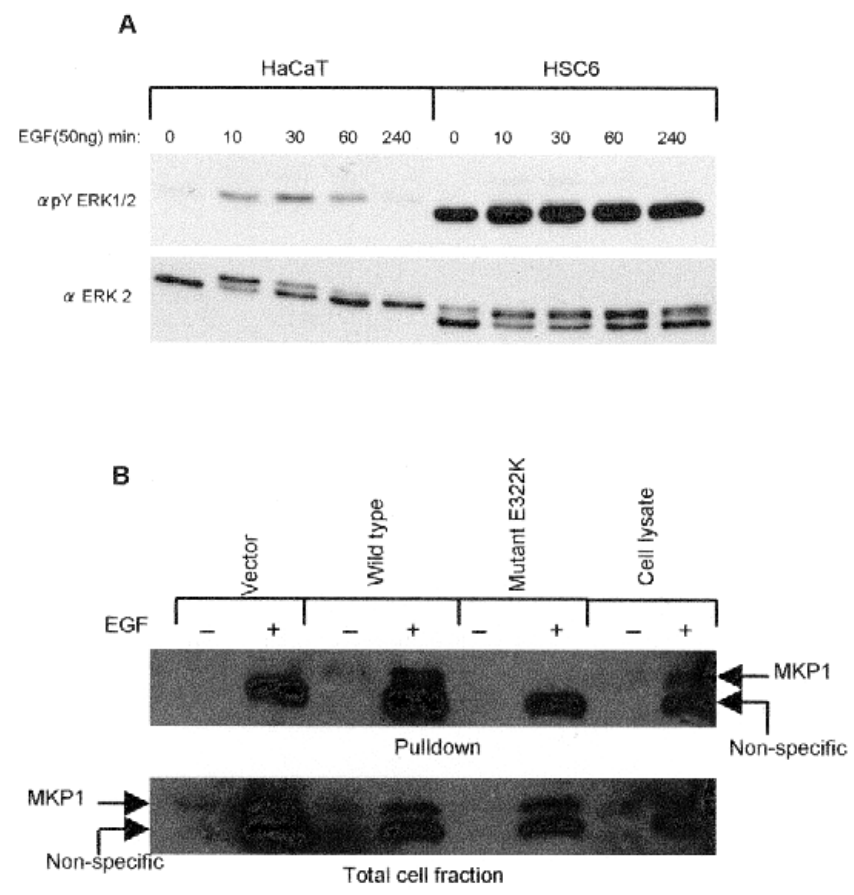

C

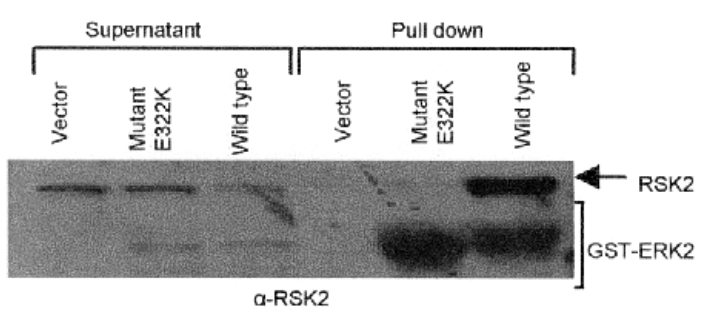

Figure 2. Biochemical and biological characteristics of $\mathrm{ERK} 22^{\mathrm{E} 322 \mathrm{~K}}$ mutation. (A) Constitutive phosphorylation of ERK2 $2^{\mathrm{E} 322 \mathrm{~K}}$ in HaCaT and HSC6 cells. Cell lysates were prepared from EGF-treated HaCaT and HSC6 cells for the indicated time period. The blotted lysates were then probed using antiphospho-tyrosine and anti-ERK2 antibodies. (B) Loss of binding of ERK2 $2^{\mathrm{E} 322 \mathrm{~K}}$ to MKP1. Cell lysates were prepared from serum-starved or EGF-treated Ca922 to induce MKP1. After pulldown with GST-ERK2 ${ }^{\text {wt }}$ or GST-ERK2 $2^{\mathrm{E} 322 \mathrm{~K}}$, the amounts of MKP1 were examined by Western blotting using anti-MKP1 antibody. (C) Loss of binding to RSK2 by pulldown assay. The binding of RSK2 to GST-ERK2 ${ }^{\mathrm{wt}}$ or GST-ERK2 $2^{\mathrm{E} 322 \mathrm{~K}}$ were examined as described in Fig. 2A, using anti-RSK2 antibody.

CD domain resulted in faster migration, whereas the mutation in the MAPK insert region did not. Not surprisingly, the ERK2 ${ }^{\mathrm{M} 13 \mathrm{~L}}$ mutant did not migrate as ERK2 ${ }^{\mathrm{E} 322 \mathrm{~K}}$ but the ERK2 ${ }^{\mathrm{M} 13 \mathrm{~L}+\mathrm{E} 322 \mathrm{~K}}$ double mutant did (Fig. 1D). It is likely that any other charge-change mutations in the CD domain of ERK2 may also result in faster migration.

ERK2 $2^{E 322 K}$ is constitutively phosphorylated and loses its interaction (in vitro) with MKP1 and RSK2. The constitutive phosphorylation of ERK2 ${ }^{\mathrm{E} 322 \mathrm{~K}}$ expressed in HSC6 cells was reported earlier (12). A time course treatment of HaCaT (an immortalized human skin keratinocyte cell line) and HSC6 cells with EGF show that the ERK2 ${ }^{\mathrm{E} 322 \mathrm{~K}}$ phosphorylation is maintained even after $4 \mathrm{~h}$ of EGF treatment. This is in contrast with the control $\mathrm{HaCaT}$ cells in which the dephosphorylation started after $30 \mathrm{~min}$ of EGF treatment (Fig. 2A). The 
A

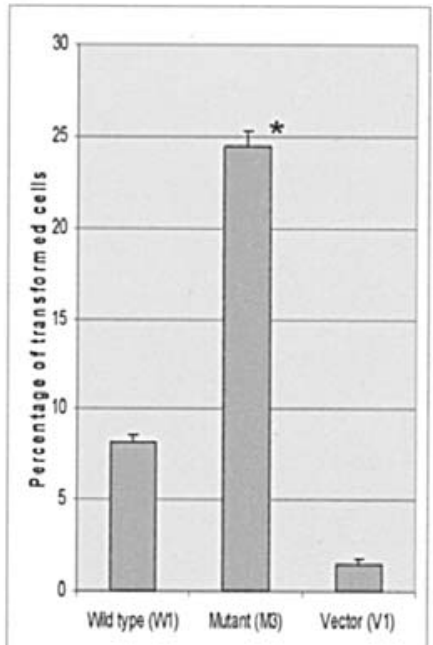

Stable Balb3T3 Clones

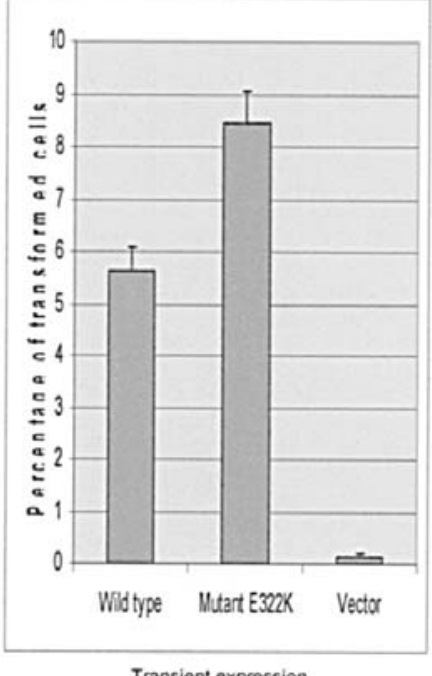

Transient expression

Figure 3. Colony forming efficiency of Balb3T3 cells in soft agar. (A) Colony formation by stable cell clones of Balb3T3 cells in soft agar. Balb3T3 cells transfected with LTR-ERK2 ${ }^{\text {wt }}$, LTR-ERK2 ${ }^{\mathrm{E} 322 \mathrm{~K}}$ or vector were G418-selected and grown as single colonies to establish stable cell clones. The clones were grown in duplicate with soft agar medium. After 4 weeks, colonies $>0.1 \mathrm{~mm}$ in size were counted and analysed. (B) Colony formation by transiently transfected Balb3T3 cells in soft agar. Balb3T3 cells were transfected with LTR-ERK2 ${ }^{\mathrm{wt}}$, LTR-ERK2 ${ }^{\mathrm{E} 322 \mathrm{~K}}$ or vector. Soft agar assay was performed as explained in Fig. 3A and statistically analysed $\left({ }^{*} \mathrm{p}=0.0035\right.$, the difference is statistically significant). The results were an average of two independent experiments.

expression of MKP1 was at its peak at $1 \mathrm{~h}$ in HSC6 cells (data not shown).

The CD domain mutation of DERK ${ }^{\text {sem }}$ was reported to have reduced binding to a number of downstream effectors which included MKP1/2/3, and RSK in vitro and in vivo (9-11). As EGF induces MKP1/2, we used EGF-treated Ca922 cell lysates to examine the interaction of MKP1 with GST-ERK2 ${ }^{\text {wt }}$ and $-\mathrm{ERK} 2^{\mathrm{E} 322 \mathrm{~K}}$. The pulldown assay showed that ERK2 ${ }^{\mathrm{wt}}$ bound to MKP1 but ERK2 ${ }^{\mathrm{E} 322 \mathrm{~K}}$ did not (Fig. 2B). The result indicates that this $\mathrm{CD}$ domain mutation of ERK2 $2^{\mathrm{E} 322 \mathrm{~K}}$ lost its capacity to bind to MKP1.

In Fig. 2C, it is evident that ERK2 ${ }^{\text {wt }}$ strongly binds to RSK2 but not to ERK2 ${ }^{\mathrm{E} 322 \mathrm{~K}}$. Thus, as seen in Fig. 2B and C, together they suggest that the ERK2 $2^{\mathrm{E} 322 \mathrm{~K}}$ mutation resulted in loss of communication with the downstream target proteins, MKP1 and RSK2, both of which were reported to contribute to the constitutive activation of ERK2.

Anchorage-independent growth of ERK2 $2^{E 322 K_{-} \text {expressing }}$ Balb3T3 cells in soft agar. As we initially found this mutation in a human cancer cell line and it was constitutively activated, we explored whether this mutant ERK2 $2^{\mathrm{E} 322 \mathrm{~K}}$ was able to enhance anchorage-independent growth. Balb3T3 cells stably (Fig. 3A) and transiently (Fig. 3B) expressing ERK2 ${ }^{\mathrm{E} 322 \mathrm{~K}}$ formed more numbers of colonies than the cells expressing wild-type ERK2 (Fig. 3A, ${ }^{*}$ p=0.0035 and B, p=0.065).

Effect of CD domain mutation DERK ${ }^{E 335 K}$ in Drosophila eye development. To delineate the physiological changes induced
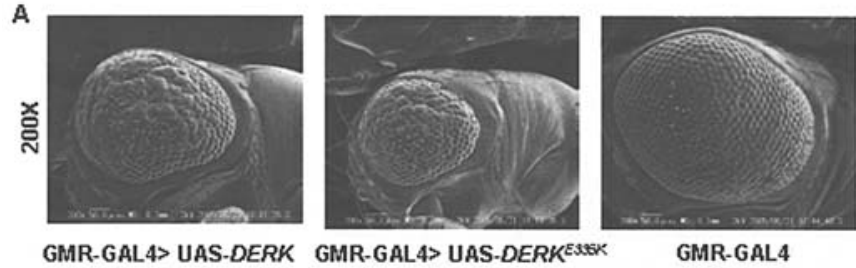

GMR-GAL4> UAS-DERK EASK

GMR-GAL4
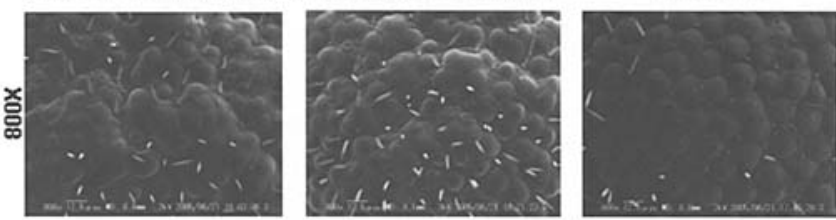

B

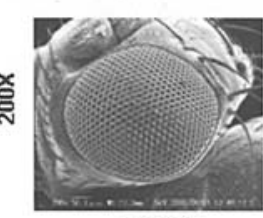

DERK ${ }^{\text {sant }}$

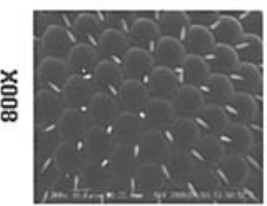

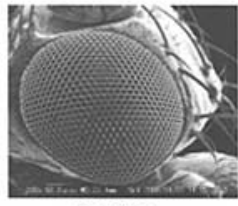

Control

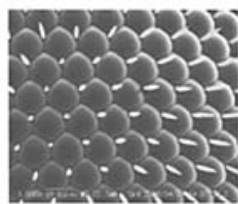

Figure 4. Drosophila transgenic for DERK ${ }^{\mathrm{E} 335 \mathrm{~K}}$ induced weakly abnormal eye development. (A) Scanning electron microscopy of the development of eyes in transgenic flies for DERK ${ }^{\mathrm{wt}}$ or DERK ${ }^{\mathrm{E} 335 \mathrm{~K}}$ or GAL4 vector only. The upper and lower panels show the $\mathrm{x} 200$ and $\mathrm{x} 800$ resolutions of the transgenic Drosophila eyes, respectively. (B) Scanning electron microscopy of the development of eyes in DERK ${ }^{\text {sem }}$ expressing fly and the control fly eye. Resolutions of $\mathrm{x} 200$ and $\mathrm{x} 800$ are shown as in A.

by the CD domain mutation in Drosophila eye development, we established transgenic Drosophila by carrying the $\mathrm{DERK}^{\mathrm{wt}}$ or the CD domain mutation DERK ${ }^{\mathrm{E} 335 \mathrm{~K}}$, corresponding to the human ERK2 ${ }^{\mathrm{E} 322 \mathrm{~K}}$. The transgenic fly was crossed with GMR-GAL4 driver strain to examine the effect of DERK ${ }^{\mathrm{E} 335 \mathrm{~K}}$ on eye development, and compared with the sevenmaker mutant DERK ${ }^{\text {sem }}$. Scanning electron microscopy of the transgenic fly for DERK ${ }^{\mathrm{E} 335 \mathrm{~K}}$ showed a more severe abnormality in adult Drosophila eye morphology than the transgenic flies for DERK ${ }^{\mathrm{wt}}$, by exhibiting a perturbed eye development with a smaller size and rougher eyes (Fig. 4A). In Fig. 4B, a comparison of the DERK ${ }^{\mathrm{sem}}$ mutant expressing fly and the control fly eye is shown.

\section{Discussion}

Our earlier report on the CD domain mutation in the human cancer cell line, HSC6, demonstrated its constitutive phosphorylation by MEK and a faster migration in SDSPAGE (12). Constitutively phosphorylated (25) and overexpressed (26) ERK had been reported in many cancers such as hepatocellular carcinomas, renal cell carcinomas, and gastric adenocarcinomas (27-29). In this study, we found that the other CD domain mutations with amino acid charge-change migrated faster and thus, may be constitutively active. Such a $\mathrm{CD}$ domain mutation was first reported as a sevenmaker mutation in Drosophila (8). 
ERK2 activity depends on the interaction with upstream and downstream effectors. The CD domain is necessary for interaction with several downstream effectors, and the MAPK insert region is important for interaction with the upstream effector, PEA-15 for nuclear entry (30). MKP1 is a phosphothreonine/tyrosine dual-specificity phosphatase, which dephosphorylates ERK1/2 in vitro and the expression of MKP1 in the cell block activation of ERK1/2 (31). In addition, ERK1/2 phosphorylates and stabilizes the MKP1 protein (32) and in turn, MKP binds to the CD domain and inactivates ERK2 (23). Our preliminary results with control HaCaT cells showed that the time course of MKP1 stimulation resulted in the decrease of ERK1/2 activation. However, this activation was not affected in HSC6 cells (Fig. 2A). Based on these results, further experiments conducted by us show that ERK2 $2^{\mathrm{E} 322 \mathrm{~K}}$ mutation lost its binding to MKP1, thus avoiding inactivation. Since it is known that MKP3 interacts with ERK2 through the CD domain, it is presumed that the interaction between them is also lost. It is known that RSK physically interacts with ERK2 and negatively regulates the nuclear entry of ERK2 (11). It was recently reported that mutant ERK2 ${ }^{\mathrm{E} 320 \mathrm{~K}}$ (rat ERK2 homolog to human ERK2 ${ }^{\mathrm{E} 322 \mathrm{~K}}$ ) is actively translocated into the nucleus as efficiently as ERK2 ${ }^{\mathrm{wt}}(30)$. Unlike the MAPK insert mutant ERK2, the CD domain double mutant D316A and D319A failed to interact to MKP3 and RSK1 in a yeast two-hybrid system showing the importance of the CD domain in the interaction of ERK2 with its cellular targets (33). It is thus suggested that loss of binding to MKP1 and RSK2 conferred to the constitutive activation on ERK2 $2^{\mathrm{E} 322 \mathrm{~K}}$, though more experiments should confirm whether this facilitated proliferation and oncogenic transformation. Our preliminary data showed three important results: i) HSC6 cells maintained phospho-threonine/tyrosine and phosphotyrosine ERK2 proteins even at $4 \mathrm{~h}$ after EGF stimulation, ii) MKP1 levels were maintained at high levels at $1 \mathrm{~h}$ after EGF stimulation and iii) serum-starved HSC6 cells (expressing ERK2 ${ }^{\mathrm{E} 322 \mathrm{~K}}$, i.e. $75 \%$ of the total ERK2 in the cell) had two times more kinase activities than the control HaCaT cells (Arvind et al, unpublished data) showing its constitutive activation by the mutant ERK2.

The constitutively active mutant ERK2 $2^{\mathrm{E} 322 \mathrm{~K}}$ is likely to present an oncogenic potential, due to loss of control with negative signaling. It should be noted that this mutation was originally identified in a human cancer cell line. These results are consistent with enhanced anchorage-independent growth by this mutation. We showed that the mutant ERK2 $2^{\mathrm{E} 322 \mathrm{~K}}$ overexpressing Balb3T3 cells induced more numbers of colonies and thus, anchorage-independent cell growth. Though the cells transiently expressing ERK2 $2^{\mathrm{E} 322 \mathrm{~K}}$ formed more numbers of colonies than those expressing wild-type (Fig. 3B), the values were not statistically significant $(p=0.065)$. However, a higher number of colonies formed by the stable cells expressing ERK2 ${ }^{\mathrm{E} 322 \mathrm{~K}}$ were statistically very significant $\left({ }^{*} \mathrm{p}=0.0035\right)$ (Fig. 3A).

The DERK ${ }^{\mathrm{E} 335 \mathrm{~K}} \mathrm{CD}$ domain mutation that we constructed, which corresponded to the ERK $2^{\mathrm{E} 322 \mathrm{~K}}$ mutation, revealed that this mutation resulted in a perturbed, smaller and rougher eye development in Drosophila. In Fig. 4B, though the external eye development of DERK ${ }^{\mathrm{D} 334 \mathrm{~N} / \mathrm{sem}}$ did not appear to be as abnormal as DERK ${ }^{\mathrm{E} 335 \mathrm{~K}}, \mathrm{DERK}^{\mathrm{D} 334 \mathrm{~N} / \mathrm{sem}}$ induced an R7 differentiation and abnormal wing formation, which were not observed with DERK ${ }^{\mathrm{E} 335 \mathrm{~K}}$ in our study (data not shown). This may be due to UAS mediating overexpression of ERK2, and inducing marked morphological changes such as small and rough eyes. Since DERK ${ }^{\mathrm{D} 334 \mathrm{~N} / \mathrm{sem}}$ is not overexpressed, it did not show such morphological abnormality. The difference in position of the mutation and the difference in the amino acid charge-change between DERK ${ }^{\mathrm{E} 335 \mathrm{~K}}$ and $\mathrm{DERK}^{\mathrm{D} 334 \mathrm{~N} / \mathrm{sem}}$ may influence the differences in inducing abnormality. It has been reported that the Drosophila ERK $^{\mathrm{D} 334 \mathrm{~N} / \mathrm{sem}}$ mutant failed to bind RSK2 (11). The importance of RSK in PC12 cell differentiation was reported earlier (34).

In summary, our results suggest that the ERK2 ${ }^{\mathrm{E} 322 \mathrm{~K}} \mathrm{CD}$ domain mutation is involved in enhanced cell growth and abnormal differentiation. Further studies of the CD domain are needed to elucidate the more novel functions of ERK2.

\section{Acknowledgements}

This work was partly supported by Grants-in-Aid from the Japan Society for the Promotion of Sciences. We are grateful to the late Dr Yajima Yoshihiro for his continuous support and valuable comments. We extend thanks to Ms. Haru Tsuchida for her financial support. MM was the recipient of the Monbukagakusho Scholarship from the Ministry of Education, Culture, Sports, Science and Technology (MEXT), Japan.

\section{References}

1. Nishida E and Gotoh Y: The MAP kinase cascade is essential for diverse signal transduction pathways. Trends Biochem Sci 18: 128-131, 1993.

2. Turjanski AG, Vaqué JP and Gutkind JS: MAP kinases and control of nuclear events. Oncogene 26: 3240-3253, 2007.

3. McKay MM and Morrison DK: Integrating signals from RTKs to ERK/MAPK. Oncogene 26: 3113-3121, 2007.

4. Ullrich A and Schlessinger J: Signal transduction by receptors with tyrosine kinase activity. Cell 61: 203-212, 1990.

5. Prowse CN, Hagopian JC, Cobb MH, Ahn NG and Lew J: Catalytic reaction pathway for the mitogen-activated protein kinase ERK2. Biochemistry 39: 6258-6266, 2000.

6. Marais R, Wynne J and Treisman R: The SRF accessory protein Elk-1 contains a growth factor-regulated transcriptional activation domain. Cell 73: 381-393, 1993.

7. Yang SH, Whitmarsh AJ, Davis RJ and Sharrocks AD: Differential targeting of MAP kinases to the ETS-domain transcription factor Elk-1. EMBO J 17: 1740-1749, 1998

8. Brunner D, Oellers N, Szabad J, Biggs WH III, Ziipursky SL and Hafen E: Again-of-function in Drosophila MAP kinase activates multiple receptor tyrosine kinase signaling. Cell 76: 875-888, 1994.

9. Chu Y, Solski PA, Khosravi-Far R, Der CJ and Kelly K: The mitogen-activated protein kinase phosphatases PAC1, MKP-1, and MKP-2 have unique substrate specificities and reduced activity in vivo toward the ERK2 sevenmaker mutation. J Biol Chem 271: 6497-6501, 1996.

10. Bott CM. Thorneycroft SG and Marshall CJ: The sevenmaker gain-of-function mutation in p42 MAP kinase leads to enhanced signalling and reduced sensitivity to dual specificity phosphatase action. FEBS Lett 352: 201-205, 1994

11. Kim M, Lee JH, Koh H, et al: Inhibition of ERK-MAP kinase signaling by RSK during Drosophila development. EMBO J 25 : 3056-3067, 2006

12. Arvind R, Shimamoto H, Momose F, Amagasa T, Omura K and Tsuchida N: A mutation in the common docking domain of ERK2 in a human cancer cell line, which was associated with its constitutive phosphorylation. Int J Oncol 27: 1499-1504, 2005.

13. Boukamp P, Petrussevska RT, Breitkreutz D, Hornung J, Markham A and Fusenig NE: Normal keratinization in a spontaneously immortalized aneuploid human keratinocyte cell line. J Cell Biol 106: 761-771, 1988. 
14. Ikeda M, Yokoyama M, Iritani A, Miura O, Katayama N, Yonemoto R, Fujinaga K, Yamaguchi $\mathrm{N}$ and Tsuchida N: Collaborative transformation with two oncogenes collaborating with $\mathrm{v}$-src in primary cells and with an immortalization-positive SV40 mutated oncogene in established cells. In: Recent Progress of Life Science Technology in Japan. Academic Press, Tokyo, 1986.

15. Dignam JD, Lebovitz RM and Roeder RG: Accurate transcription initiation by RNA polymerase II in a soluble extract from isolated mammalian nuclei. Nucleic Acids Res 11: 1475-1489, 1983.

16. Zhang S, Fukushi M, Hashimoto S, et al: A new ERK2 binding protein, Naf1, attenuates the EGF/ERK2 nuclear signaling. Biochem Biophys Res Commun 297: 17-23, 2002.

17. Huang L, Gotoh N, Zhang S, Shibuya M, Yamamoto T and Tsuchida N: SNT-2 interacts with ERK2 and negatively regulates ERK2 signaling in response to EGF stimulation. Biochem Biophys Res Commun 324: 1011-1017, 2004.

18. Huang L, Watanabe M, Chikamori M, Kido Y, Yamamoto T, Shibuya M, Gotoh N and Tsuchida N: Unique role of SNT-2/ FRS2/FRS3 docking/adaptor protein for negative regulation in EGF receptor tyrosine kinase signaling pathways. Oncogene 25: 6457-6466, 2006

19. Brand AH and Perrimon N: Targeted gene expression as a means of altering cell fates and generating dominant phenotypes. Development 118: 401-415, 1993.

20. Spradling AC: P-element-mediated transformation. In: Drosophila: A Practical Approach. Roberts DB (ed). IRL Press, Oxford, 1986.

21. Robertson HM, Preston CR, Philips RW, Johnson-Schlitz DM, Benz WK and Engels WR: A stable genomic source of P-element transposase in Drosophila melanogaster. Genetics 118: 461-470, 1988.

22. Yamaguchi M, Hirose F, Inoue YH, Shiraki M, Hayashi Y, Nishi Y and Matsukage A: Ectopic expression of human p53 inhibits entry into S-phase and induces apoptosis in the Drosophila eye imaginal disc. Oncogene 18: 6767-6775, 1999.

23. Tanoue T, Adachi M, Moriguchi T and Nishida E: A conserved docking motif in MAP kinases common to substrates, activators and regulators. Nat Cell Biol 2: 110-116, 2000.
24. Whitehurst AW, Robinson FL, Moore MS and Cobb MH: The death effector domain protein PEA-15 prevents nuclear entry of ERK2 by inhibiting required interactions. J Biol Chem 279: 12840-12847, 2004

25. Hoshino R, Chatani Y, Yamori T, et al: Constitutive activation of the 41-/43-kDa mitogen-activated protein kinase signaling pathway in human tumors. Oncogene 18: 813-822, 1999.

26. Mishima K, Inoue $\mathrm{K}$ and Hayashi $\mathrm{Y}$ : Overexpression of extracellular-signal regulated kinases on oral squamous cell carcinoma. Oral Oncol 38: 468-474, 2002.

27. Schmidt CM, McKillop IH, Cahill PA and Sitzmann JV: Increased MAPK expression and activity in primary human hepatocellular carcinoma. Biochem Biophys Res Commun 236: 54-58, 1997.

28. Oka H, Chatani Y, Hoshino R, Ogawa O, Kakehi Y, Terachi T, et al: Constitutive activation of mitogen-activated protein (MAP) kinases in human renal cell carcinoma. Cancer Res 55: 4182-4187, 1995.

29. Bang YJ, Kwon JH, Kang SH, Kim JW and Yang YC: Increased MAPK activity and MKP-1 overexpression in human gastric adenocarcinoma. Biochem Biophys Res Commun 250: 43-47, 1998.

30. Yazicioglu MN, Goad DL, Ranganathan A, Whitehurst AW, Goldsmith EJ and Cobb MH: Mutations in ERK2 binding sites affect nuclear entry. J Biol Chem 282: 28759-28767, 2007.

31. Kondoh K and Nishida E: Regulation of MAP kinases by MAP kinase phosphatases. Biochim Biophys Acta 1773: 1227-1237, 2007.

32. Brondello JM, Pouyssegur J and McKenzie FR: Reduced MAP kinase phosphatase-1 degradation after p42/p44MAPKdependent phosphorylation. Science 286: 2514-2517, 1999.

33. Robinson FL, Whitehurst AW, Raman M and Cobb MH: Identification of novel point mutations in ERK2 that selectively disrupt binding to MEK1. J Biol Chem 277: 14844-14852, 2002.

34. Silverman E, Frödin M, Gammeltoft S and Maller JL: Activation of p90 Rsk1 is sufficient for differentiation of PC12 cells. Mol Cell Biol 24: 10573-10583, 2004. 